\title{
The Promise of Biological Markers as Prognostic Determinants in Colorectal Cancer
}

\author{
Patrick G. Johnston \\ Centre for Cancer Research and Cell Biology, Queen's University Belfast, Belfast City Hospital, Northern Ireland
}

Colorectal cancer is the second main cause of cancer-related death in the Western world. Approximately $75 \%$ of patients with colorectal cancer present with localised disease. However, despite curative surgery around $40 \%$ of patients still experience disease relapse leading to morbidity and eventual mortality.

In the postoperative setting there is clear evidence that adjuvant chemotherapy significantly improves clinical outcomes in patients with colorectal cancer. Chemotherapeutic drugs such as fluoropyrimidines, oxaliplatin and irinotecan are now used as part of standard care and the arsenal of new therapies that have significant activity in this disease is steadily growing. However, the management of patients with potentially curative, locally advanced disease (stage II and III), remains an active area of clinical debate as the overall combined 5-year survival for these patients is $65 \%$ and indeed only one third of the $40 \%$ of patients who are at risk of relapse derive any benefit from adjuvant chemotherapy treatment.

Current staging and risk stratification methods in colorectal cancer, while helpful, have failed to adequately predict clinical aggressiveness and/or response to specific treatments. Our increasing knowledge of cancer biology has generated, and is promising, the development of marker candidates for more accurate prognosis assessment and therapeutic targeting in this disease. However, to date there has been no focused effort to apply these exciting discoveries to maximise patient benefit in well-designed clinical trials that assess the utility of these biological markers in clinical practice. In this issue of OnKOLOGIE, Grabowski and colleagues [Grabowski et al.: Onkologie 2005;28:399-403] present their results of a small pilot study examining the prognostic value of multi-marker analysis in stage III colorectal cancer. In this study they analyse 5 prognostic markers, including neuroendocrine differentiation, over-expression of the SIALYL-LEX antigen, over-expression of the peripheral benzodiazepine receptor (PBR), BAX protein expression and p53 mutational status.

The investigators tested each individual prognostic variable initially alone, applying a discriminant mathematical technique and were able to predict the further course of disease correctly in $55.3-70.8 \%$ of cases. However, of note, when they combined all 5 prognostic variables, the predictive value rose to $77.1 \%$ using the discriminant analysis modelling. The investigators further examined the power of the model set of 5 prognostic markers to determine risk of progression of disease in 19 separate colorectal tumours and found that the 5 marker set correctly predicted for the course of the disease in $73.6 \%$ of cases. The overall sensitivity of the multivariate discriminant analysis was $70.8 \%$ and the specificity $83.3 \%$. The conclusion from the study was that multi-marker analysis would appear to be superior to analysis of one biological variable in predicting the course of patients treated with colorectal cancer.

This study is one of an increasing number of studies examining the ability of biological variables to prognosticate for clinical outcome in patients with colon cancer. While this study clearly demonstrates the potential for various markers to be of value as prognostic indicators, and that combining prognostic variables increases the prognostic potential, there are several factors that one must consider in this study. First of all the study involved a limited number of patients $(n=49)$ where both the test set and training set were somewhat limited in size. Similarly, the confidence intervals and statistical power to define the significance of these variables is not presented which overall is a major weakness of the current study. Nonetheless, the finding that multiple markers may have the potential to be of use in correctly predicting clinical outcome for patients, is important and in line with the majority of the literature published in this area to date.

\begin{tabular}{ll}
\hline KARGER & @ 2005 S. Karger GmbH, Freiburg \\
Fax +497614520714 & Accessible online at: \\
$\begin{array}{l}\text { E-mail Information@Karger.de } \\
\text { www.karger.com }\end{array}$ & www.karger.com/onk
\end{tabular}




\section{Conclusion}

Our current staging and risk stratification methods for colorectal cancer incompletely predict prognosis and/or treatment efficacy. As new therapeutic options emerge in this disease it will be desirable, and indeed critical, to use our increasing knowledge of tumour molecular biology to optimise and individualise therapy. No biological markers have been developed to the point of allowing reliable use in colorectal cancer patients. The lack of a disciplined and focused approach to the development of these markers has resulted in insufficient scientific evidence to allow them to be introduced into routine clinical practice. The development of any potential prognostic marker for clinical use must be explored by retrospective studies such as those presented in this issue of OnKOLOGIE. When this is presented alongside complete data on routinely used factors such as histopathological grade and stage, a more convincing and complete picture is presented. Any marker that is independently associated with outcome after adjusting for such variables, may then be considered as promising and one that should be taken forward for further study of its clinical utility. Indeed the next generation of clinical studies must be designed in a manner that will test the prognostic value of defined marker sets and more precisely define not only their prognostic value but also their ability to predict the benefit of any therapeutic approach for patients with colorectal cancer. 